\title{
CAFFEINE-INDUCED POTENTIATION OF TWITCHES IN FROG SINGLE MUSCLE FIBER
}

\author{
Toshio YAMAGUCHI \\ Department of Biology, Division of Natural Sciences, \\ College of Liberal Arts, International Christian University, \\ Mitaka, Tokyo 181, Japan
}

\begin{abstract}
To clarify the detailed mechanism of caffeine-induced twitch potentiation in single muscle fibers isolated from semitendinosus muscles of the frog, the fibers were stimulated by the different patterns of repetitive pulses. On rapid application of $1 \mathrm{~mm}$ caffeine to the fiber, progressive potentiation of the twitches were observed in all cases, and as the stimulation frequencies were increased (from 0.1 to $5 \mathrm{~Hz}$ ), the periods taken to the fully potentiated twitch were shortened from $23 \mathrm{sec}$ to about $4 \mathrm{sec}$ on the average. If the repetitive stimulations at $2 \mathrm{~Hz}$ were stopped for a while during the potentiating phase of an ordinarily stimulated fiber, the fully potentiated twitch occurred later than that observed in the ordinarily stimulated fiber. It was confirmed that caffeine did not have any significant effects on both the features of the action potential and the magnitude of the resting potential of the single muscle fiber. These results indicate that the responses of the fiber themselves play an important role in the caffeine-induced twitch potentiation, and its mechanism was discussed with reference to the behavior of the fiber membrane and the triadic junction through which caffeine is assumed to act on the sarcoplasmic reticulum.
\end{abstract}

Caffeine in subcontracture concentration augments the twitch tension in frog skeletal muscle fibers without any detectable changes in the electrical responses (SANDow et al., 1964). This potentiation has been explained by the increase in the ionized calcium concentration within the fiber induced by caffeine, which may release calcium from the sarcoplasmic reticulum and also inhibit the rate of the uptake of calcium by the sarcoplasmic reticulum (WEBER and HERZ, 1968; ISAACSON and SANDOW, 1967).

Hodgkin and Horowicz (1960) and Matsumura (1967) reported that the twitch tensions of the single muscle fiber in response to repetitive stimuli increased

Received for publication June 2, 1975

山口俊夫 
progressively on rapid application of potentiator substance such as nitrate ion or caffeine. HodgKIN and Horowicz (1960) suggested that the transverse tubular system ( $T$-system) might be responsible for the progressive potentiation of the twitches, and MATSUMura (1967) has explained this as a result of caffeine diffusion into the $T$ tubules. This explanation is supported by the facts that the $T$ tubules make network connections through the cross-section of the fiber at the level of the $\mathrm{z}$ bands and have many contacts with the terminal cisternae of the sarcoplasmic reticulum. The apposing part of both membranes, the triadic junction, has a special structure consisting of 'feet' which join the both membranes at periodic intervals. In addition, the tubules have many openings, each several fibrils apart, circumferentially to the external surroundings, and a large molecular substance such as ferritin or a fluorescent dye can steadily diffuse into or out of the lumen of the tubules (Franzini-Armstrong, 1970; Peachey and Adrian, 1973; HuXleY, 1964; ENDO, 1966). Considering these studies, the most probable explanation for the twitch potentiation induced by caffeine is given as follows: caffeine might have easy access to the sarcoplasmic reticulum diffusing into the $\mathrm{T}$ tubules and consequently increase the intracellular ionized calcium concentration, which in turn augments the twitches. But, the potentiation mechanism does not seem so simple, because, as in the whole sartorius muscle of frog (SANDOW and BRUST, 1966), YAMAGUCHI (1968) has found that even in the single muscle fiber of frog, a considerable lapse of time was necessary for the fully potentiated twitch after the application of caffeine.

The author has recently found some interesting modes of caffeine-induced potentiation of twitches in single muscle fibers in response to the different patterns of repetitive stimuli. The present paper, therefore, reports these results, which will give information about its mechanism.

\section{MATERIALS AND METHODS}

Single muscle fibers isolated from the semitendinosus muscles of Rana nigromaculata were used in most experiments, and in winter the same fibers of $R$. japonica were used exclusively. The diameters of the fibers ranged from 70 to $110 \mu \mathrm{m}$. For the study of electrical responses, several fiber-bundle preparations dissected from the same muscles were used.

The bathing medium used was a Ringer solution containing (mM); $\mathrm{NaCl}, 120$; $\mathrm{KCl}, 2 ; \mathrm{CaCl}_{2}, 1.8$, buffered at $\mathrm{pH} 7.0-7.2$ with $\mathrm{NaHCO}_{3}$. An experimental solution was prepared by the addition of $1 \mathrm{mmol}$ of caffeine per liter of Ringer solution. This amount of the alkaloid is optimal to potentiate the twitches in the frog skeletal muscle without inducing any contracture (SANDow, 1965). All the fluids contained $1 \times 10^{-5} \mathrm{~g} / \mathrm{ml} \mathrm{D}$-tubocurarine chloride.

This work was divided into two parts and each part of the experiments was carried out with a different method, respectively. But, throughout the experi- 
ments, for recording the twitch tensions, the single muscle fibers were mounted horizontally in respective troughs, grooves cut in acrylite blocks, by clamping one tendon, and at the other by hooking it onto an isometric lever $(4 \mathrm{~cm})$ connected to an anode pin of a mechano-electronic transducer (RCA 5734). The fibers were stimulated by repetitive square pulses via a pair of $\mathrm{Ag} \cdot \mathrm{AgCl}$ wire electrodes which were set transversely at the bottom of the troughs. The strength of the stimulus was about twice that of the threshold and its duration was $0.5 \mathrm{msec}$. The transduced twitch tensions were fed into a dc amplifier of an oscilloscope (V C-7, Nihon Kohden Kogyo Co.) and monitored. In the first experiments, the tensions were photographed directly, whereas in the second, they were at first recorded on a magnetic tape by means of both the amplifier and a magnetic recorder (DTR 1204, Ikegami Tsushin Co.), and then photographed when played back to the oscilloscope.

In the first experiments, the fiber was stimulated in a flowing fluid which was poured into the trough with a $0.7 \mathrm{ml}$ content at a rate of $2 \mathrm{ml}$ per sec, and sucked out from the other end of the trough. In order to exchange the bathing medium rapidly, one flowing fluid was switched to another by a triple tap in the first experiments, whereas in the second, for both application and withdrawal of the bathing medium, $2 \mathrm{ml}$ of fluid was flushed into the trough with a $0.4 \mathrm{ml}$ content using a syringe in about $1.5 \mathrm{sec}$. In the latter case the excess fluid could be easily drawn out, for the surface of the trough surrounding was cut in a slope.

The stimulating pulses were generated by electronic stimulators. In the first experiments repetitive pulses of $0.1,0.2,0.5,1,2,3$, and $5 \mathrm{~Hz}$ were delivered, whereas in the second, the pulses of $2 \mathrm{~Hz}$ were from a multi-usable stimulator (MSE 40, Nihon Kohden Kogyo Co.), which could cease the train pulses at an appropriate time for a period with the aid of a micro-switch set on the flushing syringe.

All experiments were performed at room temperature $\left(18.5-28^{\circ} \mathrm{C}\right)$.

\section{RESULTS}

\section{Mechanical responses}

The experiments were first designed to test whether the rapid exchange of the solutions were completed by the above-mentioned methods. To do this, the bathing medium was exchanged by the respective methods for choline Ringer solution which contained $120 \mathrm{~mm}$ choline chloride instead of $120 \mathrm{~mm}$ sodium chloride of the normal Ringer solution. In both switching and flushing methods, about $3 \mathrm{ml}$ of the fluid was applied to a unit volume of trough contents per second, and Fig. 1 shows that the exchange of the solution was sufficiently rapid.

1) Modes of caffeine-induced potentiation of twitches in response to repetitive stimulations of different frequencies. While single muscle fibers were stimulated at $0.1,0.2,0.5,1,2,3$, and $5 \mathrm{~Hz}$, respectively, caffeine containing solution 


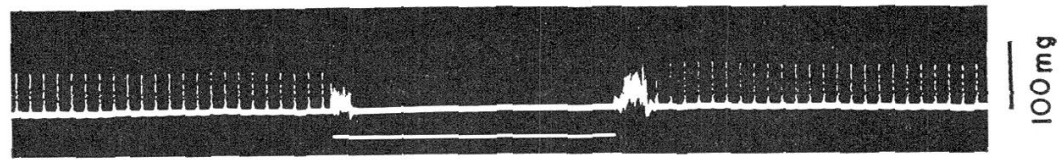

Fig. 1. Test of fluid exchange by flushing with a syringe. The fiber is stimulated at $2 \mathrm{~Hz}$ at $25^{\circ} \mathrm{C}$. Lower line indicates the bathing period with choline Ringer. The mechanical disturbances indicate the flushing periods.

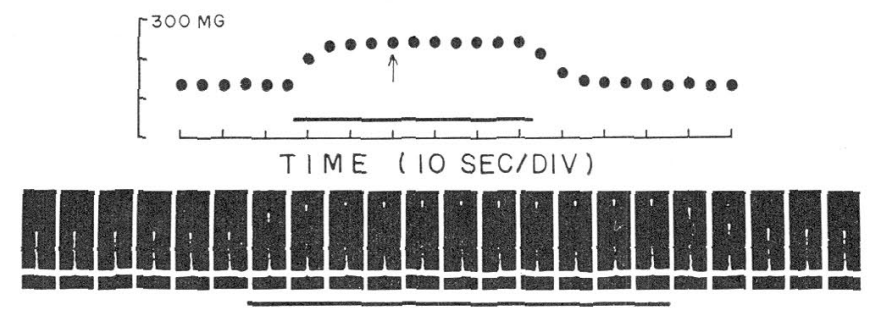

Fig. 2. Twitch potentiation by $1 \mathrm{~mm}$ caffeine in single muscle fiber stimulated in the flowing fluids at $0.2 \mathrm{~Hz}, 27.5^{\circ} \mathrm{C}$. The drawing is the illustration of the photograph, indicating the peak tensions of twitches. Arrow points the maximally developed tension. Horizontal lines indicate the bathing period with $1 \mathrm{~mm}$ caffeine.

was switched as quickly as possible after a stimulus. Then, in all cases, in consistency with the results of other authors (MATSUMURA, 1967; LÜTTGAU and OETLIKER, 1968), a staircase potentiation of twitches took place. This progressive potentiation of the twitches continued approximately for constant periods for respective frequencies of stimulations (Fig. 3), and the developed twitch tensions attained maximum values of 140 to 200 percent of the initial tensions. When the bathing solution was restored to the normal Ringer solution, twitches declined progressively and attained a constant tension level. Figure 2 illustrates a typical example of the progressive potentiation of twitches induced by caffeine in the flowing fluids.

The typical mode of twitch potentiation was sometimes obscured by fatigue and an extremely prolonged phase of progressive potentiation. Since both phenomena were frequently observed in winter frogs, the data in this section were cited from the experiments done from May to September.

The most prominent finding in the experiments was the response number attaining fully potentiated twitch in the caffeine containing solution. If the twitch is potentiated by the elevation of the caffeine concentration within the lumen of the $\mathrm{T}$ tubules due to the simple diffusion of the alkaloid, the times to the fully potentiated twitch should be equal irrespective of the stimulation frequencies, or the response number attaining maximum should be in proportion to the stimulation frequency. But, contrary to this expectation, it was revealed that the times to the fully potentiated twitches were shortened and converged at around $4 \mathrm{sec}$ as the stimulation frequencies increased. The average number of the potentiating 
twitches up to maximum and their times calculated from the response numbers are illustrated in Table 1 and Fig. 3, respectively. The number of the twitches were obtained by counting the peak tensions which were drawn from the photographs.

Table 1. Mean number of potentiating responses up to the fully developed twitch tensions in single muscle fiber of frogs on rapid application of $1 \mathrm{~mm}$ caffeine $\left(18.5-28^{\circ} \mathrm{C}\right)$.

\begin{tabular}{ccc}
\hline $\begin{array}{c}\text { Stimulation frequency } \\
(\mathrm{Hz})\end{array}$ & $\begin{array}{c}\text { Mean number of responses } \\
( \pm \text { standard error of mean) }\end{array}$ & Number of data \\
\hline 0.1 & $2.3 \pm 0.21$ & 6 \\
0.2 & $4.4 \pm 0.38$ & 8 \\
0.5 & $6.2 \pm 0.37$ & 5 \\
1 & $8.6 \pm 0.28$ & 25 \\
2 & $9.5 \pm 0.93$ & 8 \\
3 & $18.0 \pm 2.01$ & 8 \\
5 & $19.5 \pm 0.93$ & 13 \\
\hline
\end{tabular}

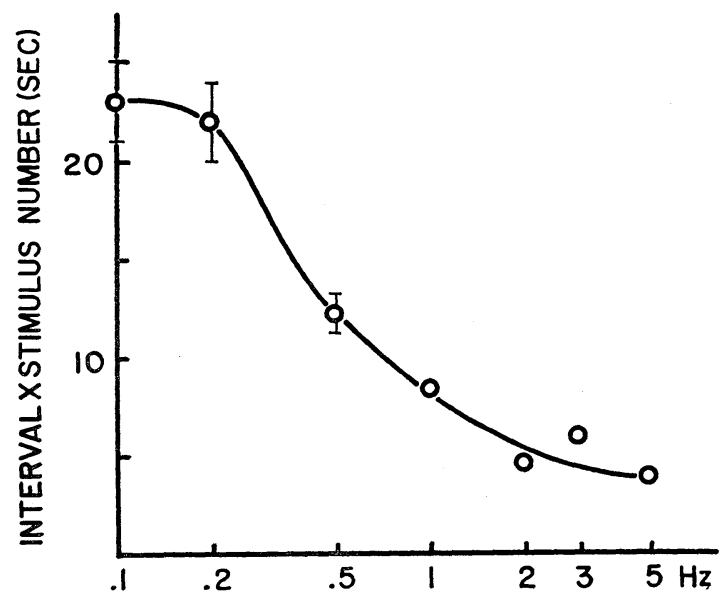

Fig. 3. The relationship between stimulation frequencies and the periods to the fully potentiated twitches in single muscle fibers calculated from the data in Table 1. Vertical bars indicate the values of stimulus interval times standard error of mean.

The twitches in response to the repetitive stimuli at the higher frequencies than $5 \mathrm{~Hz}$, were potentiated not only by the caffeine effect (RITCHIE, 1954), but also, as its result, by the summation of the twitch with the preceding relaxation phase. This phenomenon, in case of $8 \mathrm{~Hz}$ stimulation, is illustrated in Fig. 4 by the prolongation of the relaxation phase and the parallel elevations of the relaxed and the peak levels. In addition, in a short time, the twitches began to decline on account of fatigue, and the plateau level of the successive peak tensions could hardly be recognized. Accordingly, it is unreasonable to compare the results of 


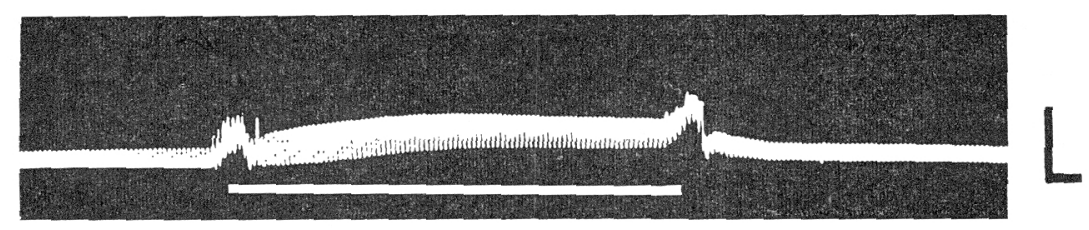

Fig. 4. Record of tension developed in single muscle fiber stimulated at $8 \mathrm{~Hz}, 22^{\circ} \mathrm{C}$. Line under the tracing indicates bathing period with $1 \mathrm{~mm}$ caffeine which applied and removed by flushing. Horizontal and vertical bars are the calibrations of $1 \mathrm{sec}$ and $200 \mathrm{mg}$, respectively.

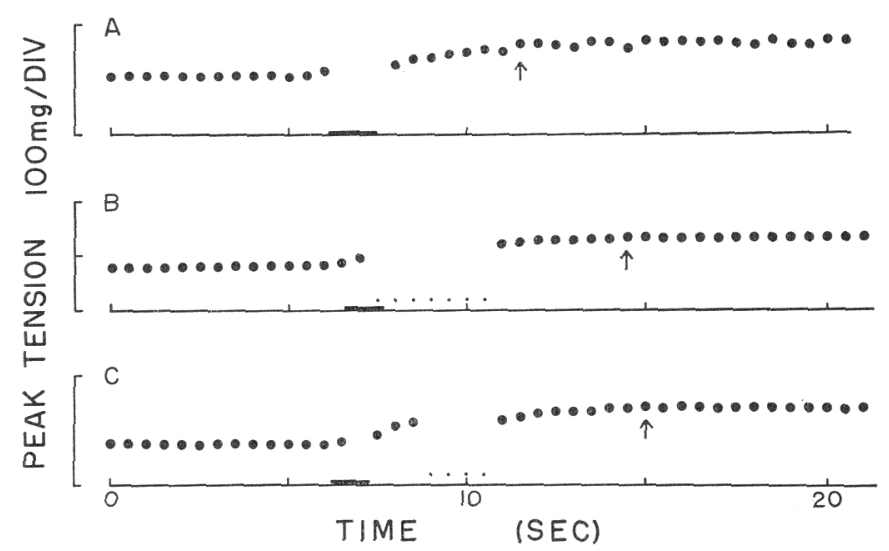

Fig. 5. Twitch potentiation by $1 \mathrm{~mm}$ caffeine in single muscle fibers stimulated by ceased train pulses at $2 \mathrm{~Hz}$. A: control experiment, $20^{\circ} \mathrm{C}, \mathrm{B}: 26.5^{\circ} \mathrm{C}, \mathrm{C}: 25^{\circ} \mathrm{C}$. Arrows indicate the responses maximally potentiated. Thick bars indicate the periods of flushing the medium, while, small dots, the omitted pulses.

the experiments at the higher frequencies than $5 \mathrm{~Hz}$ with those of other experiments.

2) Modes of caffeine-induced potentiation of twitches in response to ceased train pulses. For the further investigation of the potentiation modes of twitches in the caffeine containing solution, the experiments were conducted to find whether the fully potentiated twitch was delayed when the repetitive stimulation was ceased for a while during the potentiating phase of an ordinarily stimulated fiber. In this section, all experiments were carried out using the second method, the main differences of which were the employment of the flushing method and a magnetic recorder, besides the different pattern of stimulation.

In the control experiment (Fig. $5 \mathrm{~A}$ ), significantly the same result was obtained as in the flowing fluids (Table 1). In Fig. 5 B, a cessation of the stimuli was begun simultaneously at the time of flushing the caffeine solution into the trough, and after omission of seven stimuli, the stimulation was resumed. In this case, a slight progressive increase in twitch tension can be recognized in several responses, although, at this time, in case of the control, the developed 
twitch tension reached almost to the maximum. The delayed potentiation is more clearly seen in Fig. 5 C. In this case, four of the stimuli were omitted with a delay of $2 \mathrm{sec}$ after flushing. In this example, the first twitch tension was larger than the last before omission, but occasionally in winter frogs, this twitch tension was less than the last as shown in Fig. 6, presumably due to the decrease of calcium releasing ability of the sarcoplasmic reticulum on account of the physiological state of the winter frog.

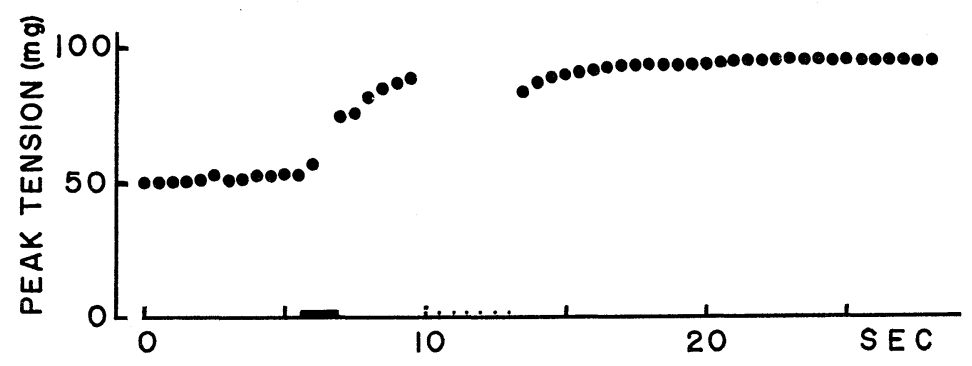

Fig. 6. Twitch potentiation by $1 \mathrm{~mm}$ caffeine in single muscle fiber stimulated by ceased train pulses at $2 \mathrm{~Hz}$. The first twitch tension after cessation is less than the last before. Thick bar on abscissa indicates the period of flushing the caffeine medium, while small dots, the omitted pulses. Experiment in January at $20^{\circ} \mathrm{C}$, Rana japonica.

\section{Electrical responses}

Resting and action potentials of the single muscle fibers were investigated by the conventional method using intracellular glass micro-electrodes filled with $2.5 \mathrm{M} \mathrm{KCl}$. As it was quite difficult to follow the action potentials with the lapse of time, recordings were finished within several minutes in the caffeine solution and the results are shown in Table 2, and, in agreement with the conclusion by SANDOw et al. (1964), confirm that neither the features of the action potential nor the magnitude of the resting potential are responsible for the potentiation of the twitches in the caffeine containing solution.

Table 2. Comparison of resting and action potentials of frog muscle fibers in $1 \mathrm{~mm}$ caffeine with those in normal solution $\left(21-22^{\circ} \mathrm{C}\right)$.

\begin{tabular}{lcccc}
\hline & Number of fibers & Resting potential & \multicolumn{2}{c}{ Action potential } \\
\cline { 3 - 5 } & & $(\mathrm{mV})$ & $(\mathrm{mV})$ & $\begin{array}{c}\text { Magnitude } \\
\text { half height } \\
(\mathrm{msec})\end{array}$ \\
\hline Control & 17 & $93.0 \pm 1.4$ & $127.5 \pm 1.5$ & $1.1 \pm 0.07$ \\
1 mM caffeine & 13 & $92.7 \pm 1.4$ & $130.7 \pm 1.7$ & $1.1 \pm 0.07$ \\
Restored & 21 & $92.9 \pm 1.2$ & $129.2 \pm 1.9$ & $1.0 \pm 0.04$ \\
\hline
\end{tabular}

\pm standard error of mean 


\section{DISCUSSION}

As mentioned in the Introduction, it is quite natural to consider that caffeine has access to the sarcoplasmic reticulum by the diffusive migration into the $T$ tubules and augments the twitches. Accordingly, for the detailed discussion of the present results, it will be necessary to calculate the saturation time of caffeine within the T-system. If the network connection of the T-system along the $\mathrm{z}$ band is assumed as an aqueous disc with a diameter of $100 \mu \mathrm{m}$ and caffeine penetrates into it from circumference only, the following equation can be applied to calculate the time taken to saturate the disc with the substance by an average of 100 percent,

$$
D t / r^{2} \fallingdotseq 1.0
$$

(HrLL, 1928),

where $D$ is the diffusion coefficient of the diffusing substance, $t$ is the time taken to saturate after application of the substance to the whole circumference and $r$ is the radius of the disc. Next, one can calculate the diffusion coefficient of a substance of a large molecule in comparison with the solvent molecule at a temperature by the following equation, which is derived from the Einstein equation,

$$
D_{T_{1}}=D_{T_{2}} \cdot T_{1} / T_{2} \cdot \eta_{T_{2}} / \eta_{T_{1}}
$$

where $D_{T_{1}}, D_{T_{2}}$ are the diffusion coefficients of the substance at the absolute temperature of $T_{1}$ and $T_{2}$, respectively and $\eta_{T_{1}}, \eta_{T_{2}}$ are the coefficients of viscosity of the solvent at $T_{1}$ and $T_{2}$. On referring to the International Critical Tables, the diffusion coefficient of caffeine in aqueous solution at $25^{\circ} \mathrm{C}$ is given as follows from equation (2),

$$
D_{25}=6.3 \times 10^{-6} \mathrm{~cm}^{2} / \mathrm{sec} .
$$

As $r=0.005 \mathrm{~cm}$, from equation (1),

$$
t \fallingdotseq 4 \mathrm{sec}
$$

which may be presumed as the saturation time of caffeine within the T-system at $25^{\circ} \mathrm{C}$, around which the experiments were performed.

BIANCHI (1962) has concluded, from his desaturation curve of radiocaffeine in muscle, that caffeine can move freely through the muscle. According to his formula fitted to the desaturation after washing out of extracellular radioactivity (KeYNes, 1954), the diffusion coefficient of caffeine at $25^{\circ} \mathrm{C}$ must be corrected to $1.38 \times 10^{-6} \mathrm{~cm}^{2} / \mathrm{sec}$, in lieu of $2.66 \times 10^{-6} \mathrm{~cm}^{2} / \mathrm{sec}$. The correct figure is reduced by a factor of 4.6 , relative to the diffusion coefficient of caffeine in aqueous phase at the same temperature. It follows, then, that caffeine moves more slowly in muscle than in aqueous medium. The real reason for the smaller diffusivity may be due to the membrane barrier and/or the physical state of the myoplasm ( $c f$. Kushmerick and Podolsky, 1969). Instead, if one puts 0.5 minute, the half- 
time of desaturation of the fast component (BIANCHI, 1962) into formula $D=$ $0.2 b^{2} / t_{1 / 2}$, (HILL, 1928), $D$ is $10.7 \times 10^{-6} \mathrm{~cm}^{2} / \mathrm{sec}$, where $t_{1 / 2}$ is the half-time of desaturation and $b$, the half-thickness of a sheet. The general agreement of this figure with the coefficient of caffeine in aqueous phase at $25^{\circ} \mathrm{C}$ confirms Bianchi's mentioning that the fast component of the desaturation curve may be ascribed to the washout of caffeine from the extracellular compartment of muscle.

On the other hand, if the diffusion coefficient of caffeine calculated from the slow component is thought of that in the muscle cell, and caffeine is assumed to penetrate the fiber from the surface membrane, the time to saturate single muscle fiber with caffeine by an average of 100 percent, is calculated at $18 \mathrm{sec}$ from formula (1), which is able to be applied to a homogeneous cylinder.

The present results show that, as the frequencies of stimulations are increased, the times to the fully potentiated twitch are shortened to the corresponding time presumably required for saturation of caffeine within the T-system at $25^{\circ} \mathrm{C}$, whereas more than $20 \mathrm{sec}$ is required at the lower frequencies of stimulation (Fig. 3). The expected time for caffeine to penetrate fully the single muscle fiber from the surface membrane is nearly consistent with that for the full potentiation only at the lower frequencies of stimulation. The results may, thus, be explained as follows: at the lower frequencies of stimulation, caffeine which diffuses into the fiber through its surface membrane, may be available, to a large extent, for the potentiation, and, at the higher frequencies of stimulation, some processes working at the fiber while it responding, play a predominant role in accelerating the potentiation by caffeine. Consequently, when the train pulses are ceased for a while during the potentiating phase of an ordinarily stimulated fiber, the times to the fully potentiated twitch are delayed as shown in Fig. 5 B, C. In addition, in these cases the twitch tensions after the cessation were much larger than before. This may be evoked, independent of the responses of the fiber, by the action of caffeine which would penetrate through the fiber membrane (BIANCHI, 1968).

As it is well known and confirmed again in the present paper that the effect of caffeine on muscle contraction is independent of the electrical events (SANDow et al., 1964), the acceleration of the potentiation by the response number may not be accounted for directly by the membrane excitation. But, it could be speculated that the entry of caffeine would be facilitated by modifications of molecular arrangement of the membrane which might be induced by membrane excitation (SINGER, 1971). In recent studies measuring the birefringence changes on isolated skeletal muscle fiber, BAYLOR and OETLIKER (1975) found a signal which precedes the signs of the excitation-contraction coupling and suggests molecular phenomena due to the action potential of the surface membrane. Although there have been no indications suggesting molecular modifications caused by fiber movement, it could not possibly be denied that the fiber movement might modify the molecular arrangement which would facilitate the entry of caffeine from the surface membrane, for the membrane structure is not so rigid (SINGER and NICOLSON, 1972). 
Next, after entering the fiber, caffeine would be distributed into the whole myoplasm more rapidly by the mechanical movement of myoplasm, due to the repetitive contraction. But, these facilitated movements of caffeine by the repetitive responses can not explain sufficiently the acceleration of potentiation, since the terminal cisternae, which is thought of as calcium storage site and ionized calcium is released from, for activation of myofilaments, are located apposing to the T-system. Therefore, it would be probable to consider that the above-mentioned caffeine acts on the longitudinal sarcoplasmic reticulum and inhibits it to accumulate the released calcium ion (BIANCHI, 1968).

As mentioned already, for the structure of the tubular system, it may be more reliable to consider that caffeine accesses easily deep into the myoplasm through the $\mathrm{T}$ tubules and acts on the terminal cisternae of the sarcoplasmic reticulum to release much more calcium ion. This is supported by the result that the times required to the fully potentiated twitches responding to higher frequencies correspond approximately to the calculated time for saturating the T-system with caffeine, while at the lower frequencies, more than $20 \mathrm{sec}$ were required (Fig. 3). This can be understood if the calcium-releasing action of the migrated caffeine into the $\mathrm{T}$ tubules is enhanced by the repetitive responses of the fiber. Thus, another explanation for the acceleration of the potentiation may be given with regard to the triadic junction. This is inferred from the work by FranzinIARMSTRONG (1970) that the narrow space separating the terminal cisternae and the $\mathrm{T}$ tubule is crossed at periodic intervals by 'feet', which join the sarcoplasmic reticulum and $\mathrm{T}$ tubule membranes. In addition, SchNeIDER and CHANDLER (1973) has suggested that the 'feet' can bear signals which transmit the tubular excitation to the sarcoplasmic reticulum. Thus, taking into account of the proposal by FuJino et al. (1972) and FujINo and FuJINo (1974) that the contractility of muscle cell may be controlled by a flexible change of the tubular system, it is speculative that diffusively migrated caffeine within the $\mathrm{T}$ tubules acts on the terminal cisternae of the sarcoplasmic reticulum through the triadic junction and this action is enhanced by each response, presumably by a gradual increase of triadic changes, functional and/or structural, induced by every repetitive activity. In addition, it is suggestive to the hypothesis, that an accommodation process in excitation-contraction coupling may take place at the $\mathrm{T}$ tubules or at their vicinity (probably at the triadic junction), of the skeletal muscle fiber in low Ca media and caffeine inhibits the increase in the rate of the accommodation process (SUGI, 1968).

As the triadic junction consists of differentiated structures such as synapses, different from the common 'cell membrane', the junction may hardly be affected by the mechanical responses of the fiber. It is now generally accepted that the tubular signal is a Na-dependent regenerative excitation similar to that of surface membrane (Costantin, 1970; Bezanilla et al., 1972; CAputo and Dipolo, 1973). In addition, recently, BezANILla and Horowicz (1975) and BAYLOR and OetLIKER (1975) have found respectively, fluorescent and birefringent signals, which are 
originated from membrane or tubular excitation, and have suggested them as signs of the depolarization of the sarcoplasmic reticulum. It might be, therefore, probable that caffeine effect is enhanced by some process due to the tubular or junctional excitation.

The author wishes to express his sincere thanks to Dr. Masahiro Fujino, Department of Physiology, Bou-Ei Medical College, for his encouragement and invaluable suggestions in preparing the manuscript. He is also indebted to Mrs. Y. Oshimi and Mr. S. Ogihara for their assistance in carrying out the experiments. This work was supported in part by a grant from the Ministry of Education.

\section{REFERENCES}

Baylor, S. M. and OetLiker, H. (1975) Birefringence experiments on isolated skeletal muscle fibres suggest a possible signal from the sarcoplasmic reticulum. Nature (Lond.), 253: 97-101.

Bezanilla, F., Caputo, C., Gonzalez-Serratos, H., and Venosa, R. A. (1972) Sodium dependence of the inward spread of activation in isolated twitch muscle fibres of the frog. J. Physiol., 223: 507-523.

Bezanilla, F. and Horowicz, P. (1975) Fluorescence intensity changes associated with contractile activation in frog muscle stained with Nile Blue A. J. Physiol., 246: 709-735.

BianchI, C. P. (1962) Kinetics of radiocaffeine uptake and release in frog sartorius muscle. J. Pharmacol. Exp. Ther., 138: 41-47.

BiANCHI, C. P. (1968) Pharmacological actions on excitation-contraction coupling in striated muscle. Fed. Proc., 27: 126-131.

CAputo, C. and Dipolo, R. (1973) Ionic diffusion delays in the transverse tubules of frog twitch muscle fibres. J. Physiol., 229: 547-557.

Costantin, L. L. (1970) The role of sodium current in the radial spread of contraction in frog muscle fibers. J. Gen. Physiol., 55: 703-715.

ENDo, M. (1966) Entry of fluorescent dye into the sarcotubular system of the frog muscle. J. Physiol., 185: 224-238.

Franzini-Armstrong, C. (1970) Studies of the triad. I. Structure of the junction in frog twitch fibers. J. Cell Biol., 47: 488-499.

Fuino, M. and Fusino, S. (1974) Mechanism of inhibition of contraction of muscle pretreated with non-electrolytes and structural basis for excitation-contraction coupling process in muscle cells. Proc. IUPS, 11: 247, New Delhi.

Fujino, M., Fusino, S., and Yamaguchi, T. (1972) Significance of transverse tubular system in determining the contractility of frog skeletal muscle. Proc. Japan Acad., 48: 539-543.

Hill, A. V. (1928) The diffusion of oxygen and lactic acid through tissues. Proc. Roy. Soc. B., 104: 39-96.

Hodgrin, A. L. and Horowicz, P. (1960) The effect of nitrate and other anions on the mechanical response of single muscle fibres. J. Physiol., 153: 404-412.

HuXLEY, H. E. (1964) Evidence for continuity between the central elements of the triads and extracellular space in frog sartorius muscle. Nature (Lond.), 202: 1067-1071.

IsAaCsON, A. and SANDOW, A. (1967) Quinine and caffeine effects on ${ }^{45} \mathrm{Ca}$ movements in frog sartorius muscle. J. Gen. Physiol., 50: 2109-2128.

Keynes, R. D. (1954) The ionic fluxes in frog muscle. Proc. Roy. Soc. B., 142: 359-382.

Kushmerick, M. J. and Podolsky, R. J. (1969) Ionic mobility in muscle cells. Science, 166: 1297-1298. 
Lüttgau, H.C. and Oetliker, H. (1968) The action of caffeine on the activation of the contractile mechanism in striated muscle fibres. J. Physiol., 194: 51-74.

Matsumura, M. (1967) Mode of action of caffeine on twitch potentiation in the frog muscle fibre. J. Physiol. Soc. Japan, 29: 170-171.

Peachey, L. D. and Adrian, R. H. (1973) Electrical properties of the transverse tubular system. In The Structure and Function of Muscle, 2nd ed., ed. by G. H. BourNe. Academic Press, New York and London, Vol. III, pp. 1-30.

Ritchie, J. M. (1954) The effect of nitrate on the active state of muscle. J. Physiol., 126: $155-168$.

SAndow, A. (1965) Excitation-contraction coupling in skeletal muscle. Pharmacol. Rev., 17: 265-320.

SANDow, A. and Brust, M. (1966) Caffeine potentiation of twitch tension in frog sartorius muscle. Biochem. Z., 345: 232-247.

SAndow, A., TAYlor, S. R., IsaAcson, A., and Seguin, J. J. (1964) Electromechanical coupling in potentiation of muscular contraction. Science, 143: 577-579.

Schneider, M. F. and Chandler, W. K. (1973) Voltage dependent charge movement in skeletal muscle: a possible step in excitation-contraction coupling. Nature (Lond.), 242: 244-246.

SINGER, S. J. (1971) The molecular organization of biological membranes. In Structure and Function of Biological Membranes, ed. by Rothfield, L. I. Academic Press, New York and London, pp. 145-222.

Singer, S. J. and Nicolson, G. L. (1972) The fluid mosaic model of the structure of cell membranes. Science, 175: 720-731.

SUGI, H. (1968) Local activation of frog muscle fibres with linearly rising currents. J. Physiol., 199: 549-567.

Weber, A. and Herz, R. (1968) The relationship between caffeine contracture of intact muscle and the effect of caffeine on reticulum. J. Gen. Physiol., 52: 750-759.

YAMAGUCHI, T. (1968). Effects of caffeine on twitch contraction elicited in frog's skeletal muscle fibres immersed in osmotically anomalous media. J. Fac. Sci. Hokkaido Univ. Ser. VI, Zool., 16: 324-338. 